\title{
Ultrafast Electron Microscopy in Materials Science
}

Wayne E, King, Michael Armstrong, Geoffrey Campbell, Alan Frank, Bryan Reed, and Brent Stuart

University of California, Lawrence Livermore National Laboratory, Livermore, CA 94550

Ultrafast optical methods have been effectively used in chemistry and biology to understand the the complex transient events that carry a reactant to a product state. More recently, $\mathrm{x}$-rays have grown in importance as an ultrafast probe and their application has been extended to materials. Applications include the study of the time evolution of phase transitions in condensed matter, gas phase chemical reactions, and the study of excited (partially unfolded) proteins, i.e., the applications cross the disciplines of chemistry, biology, and materials science. The observation of intermediate states is of particular importance to gain insight to reaction or transformation pathways. Much less attention has been given to approaches based on laser induced and electron interrogation methods, despite the fact that electron sources are brighter and their interactions with matter stronger. Use of electrons as probes has demonstrated effectiveness and great potential to study complex transient events not only because of the possible high temporal resolution, using ultrafast electron diffraction (UED), but also the potential for high spatial resolution imaging using what we have called dynamic transmission electron microscopy (DTEM) and what has previously been called high speed electron microscopy.

In this presentation, we discuss progress toward the development of a DTEM at Lawrence Livermore National Laboratory. The topics that are treated include: (1) The physics of electrons as an ultrafast probe. This encompasses the propagation dynamics of the electrons (space-charge effect, Child's law, Boersch effect) and extends to relativistic effects. (2) The anatomy of the DTEM instrument (See Figure 1). This includes discussions of the photoactivated electron gun (also known as photogun or photoelectron gun) at conventional energies (60-200 keV) and extends to $\mathrm{MeV}$ beams generated by rf guns. (3) The science of the electron detector. Various detection schemes are compared and contrasted. The effect of various physical phenomena on detective quantum efficiency (DQE) is discussed. (4) Practical aspects of operation. This includes determination of time-zero, measurement of pulse-length, strategies for pulse compression. (5) Current and potential applications in materials science, biology, and chemistry.

UEM has the potential to make a significant impact in future science and technology. Understanding of reaction pathways of complex transient phenomena in materials science, biology, and chemistry will provide fundamental knowledge that will drive new discovery-class science.

This work was performed, in part, under the auspices of the U.S. Department of Energy by the University of California, Lawrence Livermore National Laboratory under contract No. W-7405-Eng-48. 


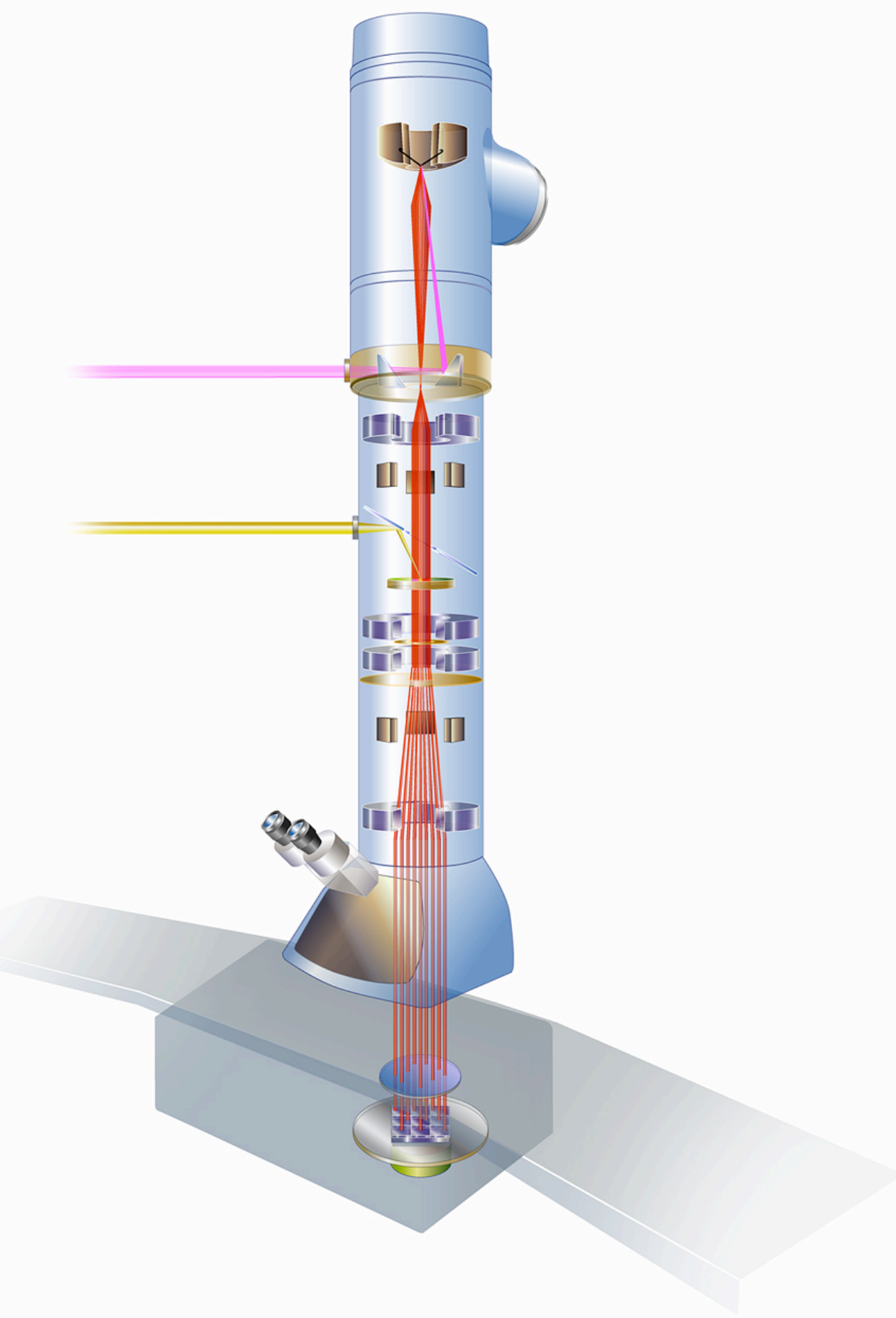

Figure 1 - Artists rendition of the LLNL DTEM. Two external lasers are introduced into the microscope column. The upper laser has a wavelength of $213 \mathrm{~nm}$ and is directed to the cathode to stimulate photoemission of the electrons. The second, lower laser has wavelength of $532 \mathrm{~nm}$ and drives a response in the specimen. 